\title{
A strong correlation exists between platelet consumption and platelet hyperactivation in COVID-19 patients. Pilot study of the patient cohort from CCH RAS Hospital (Troitsk)
}

\author{
by Stepanyan M.G. ${ }^{1,2,3}$, Martyanov A.A. ${ }^{1,2,4}$, An O.I. ${ }^{1,5}$, Boldova A.E. ${ }^{1}$, Rumyantsev S.A. ${ }^{6}$, \\ Rumyantsev A.G. ${ }^{2}$, Panteleev M.A. ${ }^{1-3}$, Ataullakhanov F.I. ${ }^{1-3}$, Sveshnikova A.N. ${ }^{1-3,5 \#}$
}

1. Centre for Theoretical Problems of Physico-Chemical Pharmacology RAS, 109029, Srednyaya Kalitnikovskaya 30, Moscow, Russia

2. Dmitriy Rogachev National Medical Research Center of Pediatric Hematology, Oncology Immunology Ministry of Healthcare of Russian Federation, 117997, Samory Machela 1, Moscow Russia

3. Lomonosov Moscow State University, Physics Faculty 119991, Leninskiye Gory 1-2, Moscow, Russia

4. N.M. Emanuel Institute of Biochemical Physics RAS (IBCP RAS), 119334, Kosygina 4, Moscow, Russian Federation

5. Federal State Autonomous Educational Institution of Higher Education I.M. Sechenov First Moscow State Medical University of the Ministry of Health of the Russian Federation, 119991, Trubetskaya 8-2, Moscow, Russia

6. Federal State Budgetary Institution of Healthcare Hospital of the Russian Academy of Sciences (Troitsk), 108840, Oktyabrski prospect 3, Troitsk, Russian Federation

\# Correspondence: a.sveshnikova@physics.msu.ru

Received: 12.06.2021

DOI: 10.52455/sbpr.01.202102011

\section{Abstract}

It is known that in COVID-19, hypercoagulation and sometimes thrombocytopenia are related to disease severity. There is also controversial data on platelet participation in COVID-19 pathology. We aimed to determine the degree of platelet hyperactivation in COVID-19 patients. Whole blood flow cytometry with Annexin- $V$ and lactadherin staining ("PS+ platelets") was utilized. Additionally, a stochastic mathematical model of platelet production and consumption was developed. Here we demonstrated that the percentage of PS+ platelets in COVID-19 patients was twofold that of healthy donors. There was a significant correlation between the amount of PS+ platelets and the percentage of lung damage in patients. No connection was found between platelet senescence and hospital therapy or patients' chronic diseases, except for chronic lung disease. Although no thrombocytopenia was observed in patients, the observed increase in platelet size (FSC-A parameter in flow cytometry) could indicate that platelet age is decreased in patients. The developed computational model of platelet turnover confirms the possibility of intense platelet consumption without noticeable changes in platelet count. We conclude that the observed platelet hyperactivation in COVID-19 could be caused by platelet activation in circulation, leading to platelet consumption without significant thrombocytopenia.

Keywords: COVID-19, platelets, coagulation, inflammation, hyperactivation
Published: 30.06 .2021

\section{Introduction}

SARS-CoV-2 is the cause of the pandemic that broke out worldwide in 2020 [1]. In patients with the severe form of the disease, there is an association with interstitial pneumonia, thrombosis, and subsequent pulmonary failure [2], [3]. Thrombocytopenia was also shown to be common in COVID-19 patients, and lower platelet count correlated with disease severity and higher mortality rates [4]-[6]. COVID-19 is known to cause a strong inflammatory response, the cytokine storm characterized by increased concentrations of interleukins $1 \beta, 6$, and 8, among others [7]. This inflammatory state can be one of the causes of serious hemostatic disorders observed in patients with severe forms of the disease [8]. Pulmonary embolism and deep vein thrombosis of the lower extremities are among the leading causes of death in COVID-19 [9]. In less severe cases, microthrombi were still present in the lungs of patients [10], [11].

The mechanism of thrombosis' initiation in COVID-19 is often proposed to be based on macrophages, the cells central to the inflammatory response in lungs [12], [13]. Macrophages, as well as lung epithelial cells, are infected by the SARSCoV-2 virions, get activated, and thus induce the inflammatory response. Consequently, the blood vessel endothelial cells also get activated [13], [14]. Activated endothelial cells express on their surface a potent activator of the plasma coagulation cascade, tissue factor (TF)[15], and secrete von Willebrand Factor (VWF), which adheres platelets to the inflamed 
endothelium [16]. Thus, COVID-19inducedendothelial cell activation results in activation of both platelet and plasma coagulation, leading to pathologic thrombosis [12]. Additional evidence for the involvement of blood coagulation in the development of COVID-19 comes from the effectiveness of treatment with heparin and its derivatives [17]. Heparins are also known for their anti-inflammatory effect [8], [18]. Although heparin effectiveness in COVID-19 treatment has been demonstrated several times [19], [20], the efficacy of the antiplatelet agents is still debatable [21].

The key driving enzyme of blood plasma coagulation, thrombin, could induce platelet hyperactivation and procoagulant response [22]. Procoagulant platelets are characterized by the presence of phosphatidylserine (PS) on their surface, thus enhancing plasma coagulation [22], [23]. PSpositive platelets were proposed to be one of the key markers of the enhanced platelet activation in the circulation [24]-[26]. It should be noted that PS+ platelets are rapidly removed from circulation by macrophages [27]. Therefore, mean platelet age can drop in case of platelet activation in circulation. It is noteworthy that younger platelets, recently produced by the megakaryocytes in the bone marrow, are larger in size [24] and more prone to activation than older platelets [28], those are sequestered in the spleen or liver after 7-10 days of circulation [25]. Thus, mean platelet size, as well as the percentage of PS+ platelets, could be indicators of platelet activation in circulation.

The mechanisms of SARS-CoV-2 influence on platelet functioning and the role of the platelet hemostasis in the pathology of COVID-19, in general, are currently actively studied. Younes et al. have shown that viral RNA was present in patients' platelets in $22 \%$ of cases, both severe and nonsevere [29]. Conversely, Manne et al. have shown that no viral RNA was found in COVID-19 patients (3 from ICU and one non-ICU) using transmission electron microscopy, while mRNA expression of the SARS-CoV-2 N1 gene was present in 2 out of 25 patients. Both of them were in ICU [3]. The angiotensin-converting enzyme 2 (ACE-2) receptor [8] is the key route for SARS-CoV-2 entrance into human cells. However, it is not clear if this receptor is present on platelets [3], [6], [29], [30]. However, ACE2 presence on platelets is controversial, as there are both works confirming [29], [31] and denying [3] it. Finally, resting platelets of COVID-19 patients were increased in size [28] and had increased expression of of P-selectin [3], [32]. However, it is noteworthy that an increase in thrombopoietin - a major inducer of platelet thrombopoiesis - has been reported in COVID-19 patients [32]. Therefore, the observed increase in platelets' size could be due to increased thrombopoientin-dependent production of the new (younger) cells [29].
To summarize the above platelets of the COVID-19 patients are defective due to reasons that are poorly understood. Here, based on the experimental observations of platelet necrosis and size, as well as computational modeling, we suggest that the observed changes in platelets may be explained by their increased activation in circulation in COVID-19 patients.

\section{Materials and Methods}

\section{Patients}

32 patients, diagnosed with coronavirus infection, who were treated at the Federal State Budgetary Healthcare Institution Hospital of the Russian Academy of Sciences (Troitsk), as well as five healthy donors, aged from 21 to 45 years, who have not been ill and have not taken any medications within the last month, were enrolled in the study. All patients' condition was described as "mild severity" by physicians and did not require mechanical ventillation. All procedures comply with the ethical standards of the National Research Ethics Committee and the 1964 Declaration of Helsinki and its subsequent amendments or comparable ethical standards. Informed voluntary consent was obtained from each of the participants included in the study. All patients were subsequently discharged from the hospital within 2 weeks due to improvement in their condition. Blood samples of five patients were analyzed twice on different days. The study was approved by the decision of the Independent Ethics Committee of the Dmitry Rogachev National Research Center No. 3/2020 dated May 19, 2020.

\section{Materials}

Annexin-Alexa647, lactadherin-FITC (Sony Biotechnology, San-Jose, USA), HEPES, bovine serum albumin (BSA), D(+)glucose (Sigma, USA); $\mathrm{NaCl} ; \mathrm{Na}_{2} \mathrm{HPO}_{4} ; \mathrm{KCl} ; \mathrm{NaHCO}_{3} ; \mathrm{MgCl}_{2} ; \mathrm{CaCl}_{2}$ (AgatMed, Moscow, Russia).

\section{Flow Cytometry}

Blood was collected in $3 \mathrm{~mL}$ tubes containing sodium citrate $(3,8 \%)$ and was kept at room temperature for 30 minutes. During that time, red blood cells sedimented into the lower layers of the blood sample. Blood was then collected from the top 10 percent of the tube's volume, was diluted in Tyrode's buffer (134 MM NaCl; $0.34 \mathrm{MM} \mathrm{Na} \mathrm{HPO}_{4}$; $2.9 \mathrm{MM} \mathrm{KCl} ; 12 \mathrm{MM} \mathrm{NaHCO} ; 20$ мM HEPES; 5 MM glucose; $1 \mathrm{MM} \mathrm{MgCl}$; $2 \mathrm{MM} \mathrm{CaCl}$; BSA $2 \%$ by weight; $\mathrm{pH} 7.3$ ) to platelet concentration of $1 \times 103$ per $1 \mathrm{~mL}$. After that, AnnexinV-Alexa647 (2\% v/v) and Lactadherin-FITC ( $2 \% \mathrm{v} / \mathrm{v})$ were added to each sample, and cells were incubated for 10 minutes, as previously described [24]. Samples were analyzed using Beckman Coulter Navios flow cytometer. The 
platelet and vesicle populations were identified by the forward and side light scattering (Fig. 1A). Annexin- $\mathrm{V}$, Lactadherin, and double-positive events were identified as shown in Fig. 1B. An event was considered PS-positive (PS+) if it was detected in lact+, anV+ or lact+anV+ gates (see Fig. 1B).

\section{Computational model}

The computational model was constructed on the principles of cellular automata models [33] when each cell is considered as an object in the program. In the model, two types of cells were present: platelets and megakaryocytes. Each platelet has two features: age and size. Each megakaryocyte has only one feature: platelet production $=\mathbf{3 5 0 0}$ (platelets per megakaryocyte, [34]). The parameter age is the number of days (model time step) for which the object exists. At each time step, $\boldsymbol{M}$ new megakaryocytes are produced in the bone marrow. $M$ is a random number from $N(\mu=10 ; \sigma=1)$, where $N\left({ }_{-} ;{ }_{-}\right)$is the normal distribution. A megakaryocyte could produce platelets only once. The model describes events related to $1 \mu \mathrm{L}$ of blood. The platelet production is also influenced by the concentration of thrombopoetin in the following manner:

$$
P=\text { PlateletProduction } \times n \times T P O,
$$

where $\boldsymbol{P}$ is the number of platelets produced by the megakaryocyte, $\boldsymbol{n}$ is the random value from $\boldsymbol{N}(\boldsymbol{\mu}=\mathbf{1} ; \boldsymbol{\sigma}=\mathbf{0 . 1})$, TPO is the parameter reflecting thrombopoietin concentration according to equation (2):

$$
T P O=1-\frac{1}{1+\left(\frac{N_{T P O}}{P l t}\right)^{h}},
$$

where $\boldsymbol{N}_{\text {TPO }}$ is the approximate number of platelets below which TPO production in the liver is initiated, and $\boldsymbol{P I} \boldsymbol{t}$ is the number of platelets at the given time point, the parameters $N_{T P O}=120000$ and $h=6$ were adjusted to describe experimental data on the relationship between platelet counts and [TPO] concentration in blood from Makar et al. [35]. In human organism platelets can be cleared from the circulation in the liver and spleen, and the probability of platelet clearance increases with platelet ageing [36]. On the model on each time step, each platelet has a removal probability:

$$
p_{\text {clear }}=n_{\text {nat }}+n_{\text {thr }},
$$

where $\boldsymbol{n}_{\text {nat }}$ reflects the natural platelet removal and is a random value from $N(0.625$ age; 0.0125 age), and $\boldsymbol{n}_{\text {thr }}$ reflects the platelets removal due to their participation in thrombus formation and is a random value from $N(0.625 \cdot K ; 0.0125 \cdot K)$, where $K$ is the consumption index, which can be varied to simulate severity of thrombosis. Platelet size is known to be related to the platelet's age and RNA content [37][39]. To simulate the distribution of platelet sizes, we have introduced equation (3) into the model, where the size parameter is recalculated from the age parameter:

$$
\text { size }=s_{0} \times\left(1-\frac{\left(1-\frac{M i n}{M a x}\right)}{1+\left(\frac{M e a n}{a g e}\right)^{h}}\right),
$$

where $s_{0}$ reflects the initial platelet size and is a value from $\mathbf{N}(\mathbf{1 2} ; \mathbf{1})$ in $[\mathrm{fL}]$, parameters $\mathbf{M a x}=\mathbf{1 1}[\mathrm{fL}]$ and Min $=\mathbf{6}[\mathrm{fL}]$ reflect the platelet size distribution found in healthy donors [40], Mean $=3$ [days] reflects median platelet age [39]. The formula (3) and coefficient $\boldsymbol{h}=$ 3 were adjusted to describe data on the relationship between the immature platelet fraction, platelet size and platelet counts [37]-[39]. The model was constructed in Python 3.7.

\section{Clinical data}

The results of complete blood count, biochemical analysis, blood clotting test, CT scans, data of daily objective examinations, age, and diagnoses of patients were kindly provided by the hospital with the patients' consent.

\section{Data processing}

Raw flow cytometry data were processed using FlowJo ${ }^{\mathrm{TM}}$ Software. Statistical analysis was performed utilizing GraphPad Prizm.

\section{Results and Discussion}

Platelets size and phosphatidylserine exposure are increased in patients with COVID-19

The patients with mild Covid-19 disease examined in this study did not suffer from thrombocytopenia, as the platelets numbers in all patients were between $139 \times 10^{3}$ and $519 \times 10^{3}$ platelets per $\mu$. To examine the size and activation status of the platelets we performed flow cytometry analyisis of cells in patients' blood stained with lactadherin and Annexin V (Fig. 1). Analysis of the platelet's forward scattering (FSA) revealed a significant increase in patients FS-A compared to healthy donors (mean value \pm SD: $213 \pm 25 \times 10^{3}$ a.u. for patients, $185 \pm 20 \times 10^{3}$ a.u. for healthy donors, $p=0.027$, Fig. 1C). These findings are in line with previously published data [3], [32]. Interestingly, Kovacz et al. have reported that increased platelet size is characteristic for patients suffering from venous thromboembolism [41], which allows us to suspect that some thrombotic events may occur in our cohort of COVID-19 patients [42]. The observed increase in platelet's size may not be exclusive for patients with SARS-COV-2 virus. A couple of recent studies have demonstrated that platelet count is significantly reduced in influenzainduced pneumonia, and the mean platelet volume is significantly increased [43], [44]. Severe thrombocytopenia is extremely rare for the EpsteinBarr virus, but this infection often presents a mild reduction in platelet counts as well as moderate 
increase in platelet size for uncomplicated cases [45].

The percentage of PS+ platelets in patients was more than two times higher than that in healthy donors (mean value \pm SD: $0.74 \pm 0.37 \%$ for patients, $0.29 \pm 0.07 \%$ for healthy donors; $p<0.0001$, Fig. 1D$\mathrm{F})$. The average percentage of PS+ events was higher for lactadherin than for annexin $\mathrm{V}(\mathrm{p}=0.0372$, Fig. 1E, F), which is consistent with the literature data on the higher effectivity of lactadherin for PS+ cells detection [46]. The values obtained for both PS+ platelet markers correlated well with each other $(r=0.64, p<0.0001)$, and the total percentage of PS+ events $(r=0.74, p<0.0001$ for annexin $V, r=0.96$, $p<0.0001$ for lactadherin, see Table 1). Therefore, we observe platelet hyperactivation in COVID-19, which is in line with previously published data [32].

The PS+ platelets are known to form in response

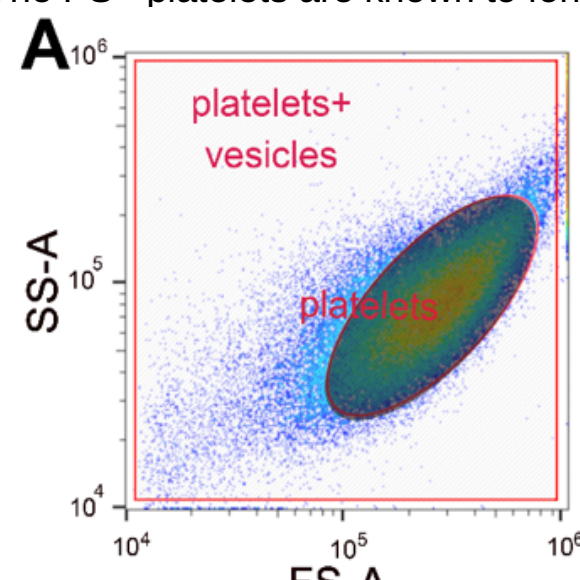

FS-A
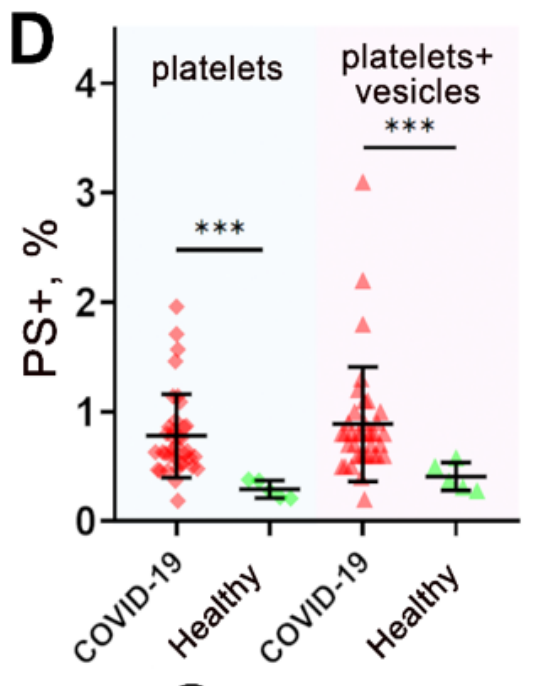

$\mathbf{G}$

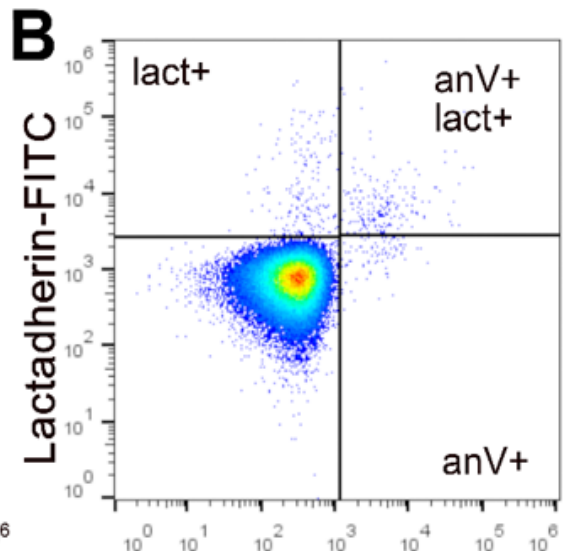

AnnexinV-Alexa 647

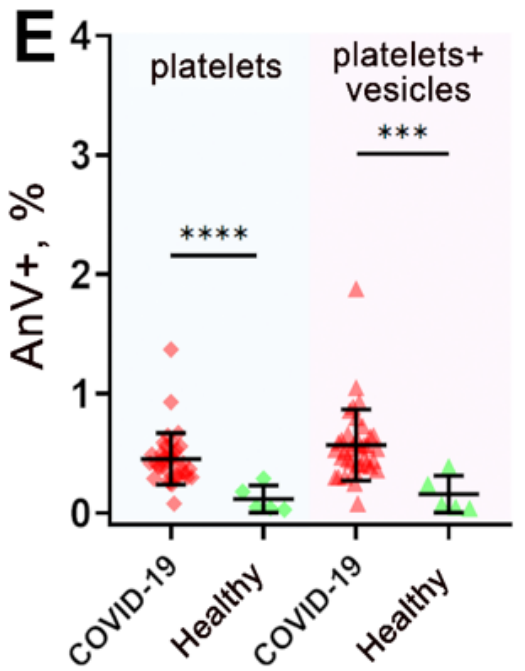

to strong stimulation, for example, dual collagen and thrombin [47], [48]. The observed increase in percentage of PS+ platelets (0.5-1.5\%) in Covid-19 patients could reflect an ongoing platelet activation in circulation [49]. An inflammatory process in the COVID-19 patient's body is inextricably linked with the activation of cells in adjacent tissues [12] and may lead to platelets' activation due to their contact with the products of thrombus-forming processes (such as the contents of platelet granules, thrombin, ADP, etc.) [3]. Thus, in case of moderate ongoing lung microthrombosis, weak increase of PS+ platelet number could be observed. This is in line with the clinical picture of the studied patients. None of them suffered from severe thrombotic complications during the course of the study. 
Correlation of platelet parameters with clinical data

Platelets are not only the critical players in coagulation but are also an essential source of inflammatory mediators. In addition to anticoagulant therapy, applied to COVID-19 patients, antiinflammatory therapy is also carried out using various approaches: these include immunosuppressants (for example, actemra), antimalarial drugs (hydroxychloroquine) and corticosteroids, IL-6 antagonists (tocilizumab) [7]. Therefore we analyzed the correlation between platelet parameters, the applied therapy, and the concurrent patients' diseases.

The proportion of PS+ platelets correlated $(r=0.69, p<0.01)$ with the lung damage (according to CT scan performed no later than 2 days from the date of platelet examination, Table 1). These results are in good agreement with the hypothesis about the influence of the inflammatory process occurring in the lungs on the coagulation system since an increase in the area of affected alveoli leads to activation of adjacent endothelial tissues [50], [51]. In addition, there is a negative correlation ( $r=-0.49$, $p<0.05)$ between the total number of platelets and the number of procoagulant ones. We presume that high consumption of platelets can cause these changes (Table 1). The percentage of PS+ platelets and platelet count correlated with blood fibrinogen levels $(p<0.05$ and $p<0.001$ respectively, Table 1$)$. This result may be explained by thrombus formation leading to increased consumption of clotting factors

\begin{tabular}{|c|c|c|c|c|c|c|}
\hline & $\mathrm{AnV}+$ & Lact + & PS+ & Age & $\begin{array}{l}\text { Lung } \\
\text { Damage }\end{array}$ & Resp. Rate \\
\hline Annexin+ & & 0.64 & 0.74 & -0.07 & 0.42 & 0.15 \\
\hline Lactadherin+ & 0.64 & & 0.96 & -0.43 & 0.71 & 0.22 \\
\hline PS+ & 0.74 & 0.96 & & -0.34 & 0.69 & 0.10 \\
\hline Platelets & -0.53 & -0.47 & -0.49 & 0.02 & -0.50 & -0.36 \\
\hline $\begin{array}{l}\text { Platelet } \\
\text { Forward } \\
\text { Scattering }\end{array}$ & 0.41 & 0.33 & 0.35 & -0.15 & 0.48 & 0.27 \\
\hline & CRP & AST/ALT & Bilirubin & Glucose & Total protein & Hemoglobin \\
\hline Annexin+ & 0.10 & -0.08 & -0.10 & -0.12 & -0.76 & 0.32 \\
\hline Lactadherin+ & 0.13 & -0.11 & -0.24 & -0.10 & -0.56 & 0.21 \\
\hline PS+ & 0.07 & -0.11 & -0.26 & -0.14 & -0.51 & 0.24 \\
\hline Platelets & -0.19 & -0.17 & -0.03 & 0.02 & 0.90 & 0.26 \\
\hline $\begin{array}{l}\text { Platelet } \\
\text { Forward } \\
\text { Scattering }\end{array}$ & 0.20 & -0.51 & 0.00 & -0.04 & -0.79 & 0.31 \\
\hline & Platelets & $\begin{array}{l}\text { Platelet } \\
\text { Forward } \\
\text { Scattering }\end{array}$ & Leukocytes & INR & Fibrinogen & D-dimer \\
\hline Annexin+ & -0.53 & 0.41 & -0.22 & 0.06 & -0.61 & 0.22 \\
\hline Lactadherin+ & -0.47 & 0.33 & -0.16 & -0.13 & -0.42 & 0.23 \\
\hline PS+ & -0.49 & 0.35 & -0.18 & -0.09 & -0.50 & 0.17 \\
\hline Platelets & & -0.29 & 0.22 & -0.14 & 0.63 & -0.29 \\
\hline $\begin{array}{l}\text { Platelet } \\
\text { Forward } \\
\text { Scattering }\end{array}$ & -0.29 & & 0.14 & -0.13 & -0.19 & 0.17 \\
\hline
\end{tabular}

Table 1. Pearson's correlation coefficients (R) for the percentage of phosphatidylserine-positive (PS+) events, platelet counts, and platelet forward scattering with indicators of the patient's condition and test values: age, percentage of lung damage (by $\mathrm{CT}$ ), respiratory rate, C-reactive protein (CRP), AST / ALT, total bilirubin, glucose, total protein, hemoglobin, platelets, platelet forward scattering, leukocytes, INR, fibrinogen, D-dimer. Green values indicate $p$-value $<0.05$. and platelets. Owing to this, the liver compensates for protein intake [28], [52]. No correlation was found between procoagulant platelets and C-reactive protein or D-dimer (see Table 1).

\section{Correlation of platelet parameters with the applied} therapy

In the hospital, all patients with suspected COVID-19 were prescribed thromboprophylaxis [53]. The use of heparins can lead to transient heparininduced thrombocytopenia (HIT) [54], [55]. However, it was not observed in our study. The amount of PS+ platelets for patients receiving heparins also was not altered compared to other COVID-19 patients (Fig. 2A). Sometimes, antiplatelet drugs are prescribed for COVID-19 patients [56]. For two out of three patients receiving antiplatelet drugs in our study because of previous prescriptions, the amount of PS+ platelets was significantly lower than for other COVID-19 patients (Fig. 2A).

According to previously published recommendations, other therapeutic agents are prescribed for the patients in the hospital, including the antiretroviral drug lopinavir [57], [58]. There were no differences in the number of procoagulant platelets between the groups of patients who took and did not take the lopinavir-ritonavir combination (Fig. 2A).

When comparing groups of patients for the presence of chronic diseases, statistically significant differences $(p=0.027)$ were found only for the group of patients with chronic lung diseases. The percentage of PS+ events in the presence of the disease was lower than in the absence (mean value \pm SD: $0.81 \pm 0.37 \%$ for absence, $0.41 \pm 0.17 \%$ for presence, Fig. 2B), which may be due to the presence of a compensatory mechanism in these patients' coagulation system. The patient cohort included in this study is highly heterogenic in terms of age and health status. However, the lack of significant differences between different patients' groups (Fig. 2B, Fig. S1) and the absence of the observed altered platelet phenotype in chronic diseases [59]-[62] allows us to claim that this phenotype is the result of COVID-19.

\section{The computational model predicts that the enhanced platelet consumption underlies thrombocytopathy in COVID-19 patients}

Platelet phenotype of COVID-19 patients appeared to be distinctive: while no significant thrombocytopenia was detected in our study as well as in the literature [3], [28], [29], [32], increased platelet size and increased PS+ platelet subpopulation were also noted. In order to understand, whether such phenotype could be caused by the consumption of platelet due to thrombosis, we have constructed the model of the platelet production, ageing, and clearance (Fig. 3A).

In the model, in the absence of additional 


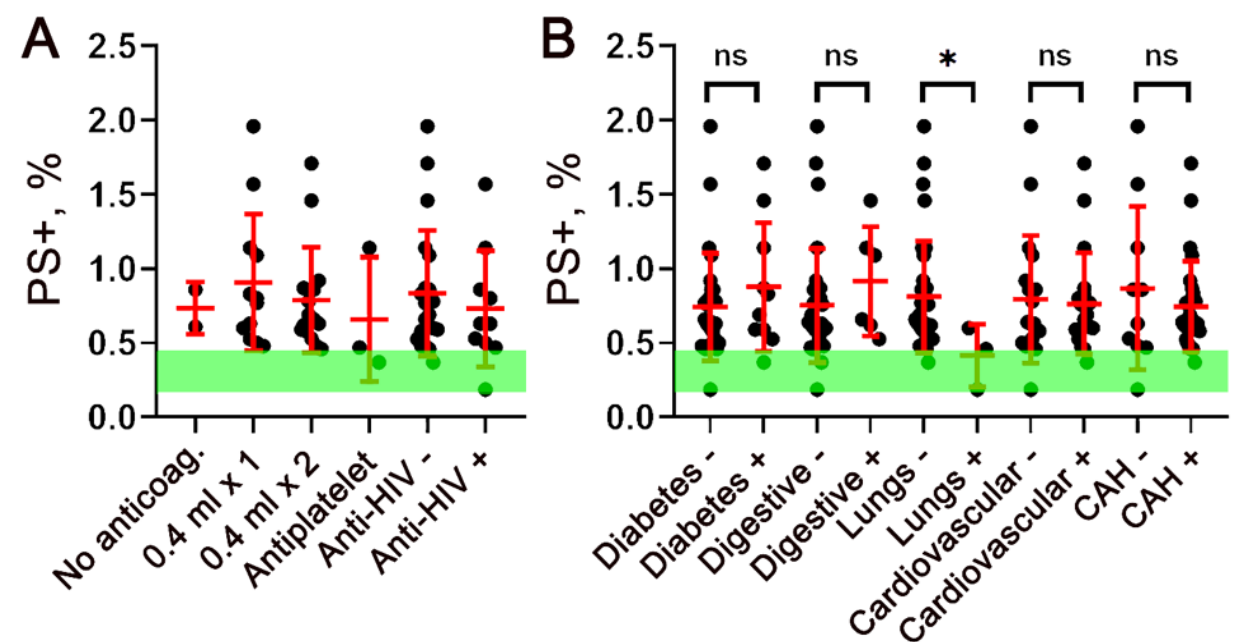

Figure 2. Association of phosphatidylserine-positive events with clinical parameters of patients, the presence of chronic diseases, and ongoing therapy. A - Comparison of the results of patients on the therapy they take (3 different groups by the amount of low molecular weight heparins, a group was taking anticoagulants and antiplatelet agents, groups taking anti-HIV drugs and not taking them). When using the Mann-Whitney criterion statistics no significant differences were found between the groups. B - Comparison of the percentage of phosphatidylserine-positive events for groups of patients suffering from various chronic diseases (type II diabetes mellitus (Diabetes), diseases of the gastrointestinal tract (Digestive), lungs (Lungs; $p=0.0274)$, cardiovascular (Cardiovascular), chronic arterial hypertension (CAH)). The green rectangle shows the normal range obtained for healthy donors (mean \pm 2 standard deviations). The MannWhitney test was used for significance; one asterisk indicates $p<0.05$.

platelet consumption, the average platelet counts are between $155 \times 103$ platelets/ $\mu$ l and $177 \times 103$ platelets/ $\mu$ l (Fig. 3B). The number of platelets and platelet size were stochastically fluctuating (Fig. S2 $A$ and $B$ correspondingly). Introduction of additional consumption, which did not significantly alter platelet counts $(\Delta \mathrm{Plt}<25000 / \mu \mathrm{l})$, results in a significant increase in the platelet size (Fig. 3B,C; S2A,B). Increased consumption also resulted in the platelets becoming younger (Fig. S2C,D). Enhancement of the platelet consumption above 2 resulted in mild thrombocytopenia and platelets becoming younger (Fig. 3B). Severe thrombocytopenia appeared at platelet consumption above $\mathrm{K}=5$, which corresponded to more than half of the produced platelets being consumed (Fig. 3B, S2). Additional sensitivity analysis revealed that model outcome was most sensitive to the TPO synthesis, platelet consumption, and platelet clearance parameters. These reactions are highlighted in red (Fig. 3A).

It is noteworthy that in patients the PS+ platelet fraction weakly correlated with the mean FS-A values
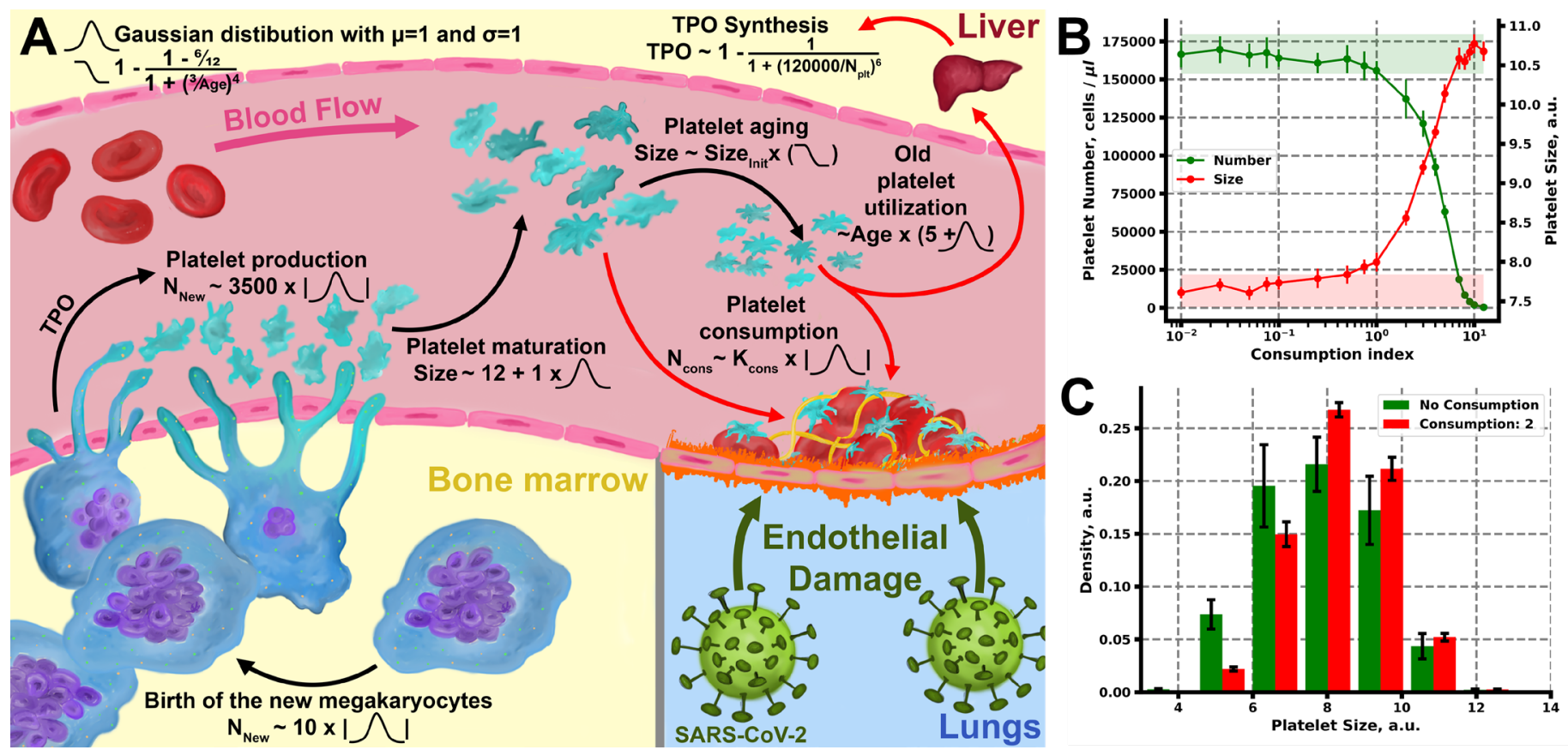

Figure 3. Computational model of platelet production in the presence of COVID-19 induced thrombosis. A Detailed scheme of the model (most sensitive reactions are highlighted in red). B - Dependence of the average platelet count (green curve and dots) and platelet size (red curve and dots) from the platelet consumption index in the model. Platelet number and size in the absence of consumption lie in the areas, highlighted by green and red rectangles correspondingly. C - Platelet size distribution in the absence (green bars) and the presence (red bars) of consumption (with consumption index set to 2). Whiskers on all plots represent SD. 
(Table 1). This finding supports the hypothesis that in COVID-19, platelets are consumed due to thrombosis which leads to the increase in PS+ platelet fraction, younger overall age of platelets and therefore larger size. The weakness of the correlation could be caused by the enhanced PS+ platelet consumption by the liver and spleen [36]. However, it should also be kept in mind that the cells were resting during the experiment, and no comparison of the degree of activation for patients and healthy donors was carried out.

\section{Conclusions}

Here we observed a significant increase in the fraction of PS+ platelets in COVID-19 patients (Fig. 1). This phenomenon does not correlate either with therapeutic interventions carried out in the hospital or with chronic diseases in the patients (Fig. 2). Therefore, we assume that the observed increase in platelet size and phosphatidylserine exposure is mainly caused by COVID-19 and associated pneumonia. Previously it has been reported that such changes could be caused by active thrombosis [26], [41]. The proposed computational model demonstrates that moderate consumption, which does not result in pronounced thrombocytopenia, could result in a 1.3-fold increase in mean platelet volume observed for the COVID-19 patients (Fig. 1).

Based on our experimental results and theoretical findings, we propose the following scheme of the COVID-19 impact on human platelets:

- SARS-CoV-2 induces lung damage. This results in the blood vascular endothelium activation and tissue factor (TF) exposure to the blood flow [12], [13];

- TF induces thrombus formation and platelet consumption;

- Fraction of PS+ platelets is increased (Fig. 1) as a result of strong platelet activation, and platelets production is enhanced by the megakaryocytes (Fig. 3) [3], [29], [32] due to the increased TPO synthesis by the liver [3];

- A mild reduction of the platelet count occurs [3], [52], while platelet size increases significantly [3], [28].

Therefore, it can be assumed that a therapeutic correction of the pro-thrombogenic state of the lung vascular endothelium could improve the platelet's quality. Still, further study of platelets and their role in the development of COVID-19 is necessary, as platelets can be used to monitor the hemostasis system in COVID-19 patients.

\section{Author Contributions}

S.M.G. performed the experiments, processed clinical and experimental data and wrote the paper, M.A.A. developed the model, processed the modeling data, performed the experiments and edited the paper, A.O.I. processed the clinical data, B.A.E. processed the experimental data, R.S.A. and R.A.G. planned the project and processed the clinical data, P.M.A. and A.F.I. planned and supervised the project, S.A.N. planned and supervised the project, processed the data, developed the model and wrote the paper. All authors provided critical feedback and helped shape the research, analysis and manuscript.

\section{Funding}

The work was supported by the Russian Presidential Scholarship SP-2675.2019.4; a grant from the endowment foundation "Science for Children" and Lomonosov Moscow State University Digital Medicine School.

\section{References}

1. Q. Li et al., 'Early Transmission Dynamics in Wuhan, China, of Novel CoronavirusInfected Pneumonia', New England Journal of Medicine, vol. 382, no. 13, pp. 1199-1207, Jan. 2020, doi: 10.1056/NEJMoa2001316.

2. C. Huang et al., 'Clinical features of patients infected with 2019 novel coronavirus in Wuhan, China', The Lancet, vol. 395, no. 10223, pp. 497-506, 2020, doi: 10.1016/ S0140-6736(20)30183-5.

3. B. K. Manne et al., 'Platelet gene expression and function in patients with COVID-19', Blood, vol. 136, no. 11, pp. 1317-1329, Sep. 2020, doi: 10.1182/blood.2020007214.

4. X. Yang et al., 'Thrombocytopenia and its association with mortality in patients with COVID-19', Journal of Thrombosis and Haemostasis, vol. 18, no. 6, pp. 1469-1472, 2020, doi: https://doi.org/10.1111/jth.14848.

5. G. Lippi, M. Plebani, and B. M. Henry, 'Thrombocytopenia is associated with severe coronavirus disease 2019 (COVID-19) infections: A meta-analysis', Clinica Chimica Acta, vol. 506, pp. 145-148, Jul. 2020, doi: 10.1016/j.cca.2020.03.022.

6. O. I. An et al., 'Platelets in COVID-19: "innocent by-standers" or active participants?', Voprosy gematologii/onkologii i immunopatologii v pediatrii, vol. 20, no. 1, pp. 184-191, Apr. 2021, doi: 10.24287/1726-1708-2021-20-1184-191.

7. Q. Ye, B. Wang, and J. Mao, 'The pathogenesis and treatment of the 'Cytokine Storm' in COVID-19.', The Journal of infection, vol. 80, no. 6 , pp. 607-613, Jun. 2020, doi: 10.1016/j. jinf.2020.03.037.

8. E. Terpos et al., 'Hematological findings and complications of COVID-19', American Journal of Hematology, vol. 95, no. 7, pp. 
834-847, Jul. 2020, doi: 10.1002/ajh.25829.

9. M. Panigada et al., 'Hypercoagulability of COVID-19 patients in intensive care unit: A report of thromboelastography findings and other parameters of hemostasis.', Journal of thrombosis and haemostasis: JTH, vol. 18, no. 7 , pp. 1738-1742, Jul. 2020, doi: 10.1111/ jth.14850.

10. N. Tang, H. Bai, X. Chen, J. Gong, D. Li, and $Z$. Sun, 'Anticoagulant treatment is associated with decreased mortality in severe coronavirus disease 2019 patients with coagulopathy', Journal of Thrombosis and Haemostasis, vol. 18, no. 5, pp. 1094-1099, May 2020, doi: 10.1111/jth.14817.

11. F. A. Klok et al., 'Incidence of thrombotic complications in critically ill ICU patients with COVID-19', Thrombosis research, vol. 191, pp. 145-147, Jul. 2020, doi: 10.1016/j. thromres.2020.04.013.

12. M. Z. Tay, C. M. Poh, L. Rénia, P. A. MacAry, and L. F. P. Ng, 'The trinity of COVID-19: immunity, inflammation and intervention', Nature Reviews Immunology, vol. 20, no. 6, Art. no. 6, Jun. 2020, doi: 10.1038/s41577020-0311-8.

13. R. Alon et al., 'Leukocyte trafficking to the lungs and beyond: lessons from influenza for COVID-19', Nature Reviews Immunology, pp. 1-16, Nov. 2020, doi: 10.1038/s41577-02000470-2.

14. Bonaventura et al., 'Endothelial dysfunction and immunothrombosis as key pathogenic mechanisms in COVID-19', Nat Rev Immunol, vol. 21, no. 5, Art. no. 5, May 2021, doi: 10.1038/s41577-021-00536-9.

15. M. Witkowski, U. Landmesser, and U. Rauch, 'Tissue factor as a link between inflammation and coagulation.', Trends in cardiovascular medicine, vol. 26, no. 4, pp. 297-303, May 2016, doi: 10.1016/j.tcm.2015.12.001.

16. F. Denorme, K. Vanhoorelbeke, and S. F. De Meyer, 'von Willebrand Factor and Platelet Glycoprotein Ib: A Thromboinflammatory Axis in Stroke', Front Immunol, vol. 10, p. 2884, 2019, doi: 10.3389/fimmu.2019.02884.

17. J. Hirsh et al., 'Heparin and Low-MolecularWeight Heparin Mechanisms of Action, Pharmacokinetics, Dosing, Monitoring, Efficacy, and Safety', CHEST, vol. 119, no. 1, pp. 64S-94S, Jan. 2001, doi: 10.1378/ chest.119.1_suppl.64S.

18. J. M. Connors and J. H. Levy, 'COVID-19 and its implications for thrombosis and anticoagulation.', Blood, vol. 135 , no. 23 , pp. 2033-2040, Jun. 2020, doi: 10.1182/ blood.2020006000.
19. C. Carallo et al., 'Higher heparin dosages reduce thromboembolic complications in patients with COVID-19 pneumonia', J Investig Med, Mar. 2021, doi: 10.1136/jim2020-001628.

20. J. Gratz et al., 'Risk of Clinically Relevant Venous Thromboembolism in Critically III Patients With COVID-19: A Systematic Review and Meta-Analysis', Front Med (Lausanne), vol. 8, p. 647917, 2021, doi: 10.3389/fmed.2021.647917.

21. V. Bianconi, F. Violi, F. Fallarino, P. Pignatelli, A. Sahebkar, and M. Pirro, 'Is Acetylsalicylic Acid a Safe and Potentially Useful Choice for Adult Patients with COVID-19 ?', Drugs, vol. 80 , no. 14 , pp. $1383-1396$, Sep. 2020, doi: 10.1007/s40265-020-01365-1.

22. A. N. Sveshnikova et al., 'Systems biology insights into the meaning of the platelet's dual-receptor thrombin signaling', Journal of Thrombosis and Haemostasis, vol. 14, no. 10, pp. 2045-2057, 2016, doi: 10.1111/jth.13442.

23. N. A. Podoplelova et al., 'Coagulation factors bound to procoagulant platelets concentrate in cap structures to promote clotting', Blood, vol. 128, no. 13, pp. 1745-1755, 29 2016, doi: 10.1182/blood-2016-02-696898.

24. A. A. Ignatova et al., 'Flow cytometry for pediatric platelets', Platelets, vol. 30, no. 4, pp. 428-437, 2019, doi: 10.1080/09537104.2018.1513473.

25. H. H. Versteeg, J. W. M. Heemskerk, M. Levi, and P. H. Reitsma, 'New fundamentals in hemostasis', Physiol. Rev., vol. 93, no. 1, pp. 327-358, Jan. 2013, doi: 10.1152/ physrev.00016.2011.

26. D. Y. Nechipurenko et al., 'Clot Contraction Drives the Translocation of Procoagulant Platelets to Thrombus Surface', Arterioscler Thromb VascBiol, vol. 39, no. 1, pp. 37-47, Jan. 2019, doi: 10.1161/ATVBAHA.118.311390.

27. M. T. G. Alonzo et al., 'Platelet Apoptosis and Apoptotic Platelet Clearance by Macrophages in Secondary Dengue Virus Infections', The Journal of Infectious Diseases, vol. 205, no. 8, pp. 1321-1329, Apr. 2012, doi: 10.1093/ infdis/jis180.

28. G. D. Wool and J. L. Miller, 'The Impact of COVID-19 Disease on Platelets and Coagulation', PAT, pp. 1-13, Oct. 2020, doi: 10.1159/000512007.

29. Z. Younes et al., 'Platelets Can Associate with SARS-Cov-2 RNA and Are Hyperactivated in COVID-19', Circulation Research, vol. 0, no. 0, Oct. 2020, doi: 10.1161/ CIRCRESAHA.120.317703.

30. J. M. Burkhart et al., 'The first comprehensive 
and quantitative analysis of human platelet protein composition allows the comparative analysis of structural and functional pathways', Blood, vol. 120, no. 15, pp. e73-e82, Oct. 2012, doi: 10.1182/blood-2012-04-416594.

31. S. Zhang et al., 'SARS-CoV-2 binds platelet ACE2 to enhance thrombosis in COVID-19', Journal of Hematology \& Oncology, vol. 13, no. 1 , p. 120,2020 , doi: $10.1186 / s 13045-$ 020-00954-7.

32. F. Taus et al., 'Platelets Promote Thromboinflammation in SARS-CoV-2 Pneumonia', Arterioscler Thromb Vasc Biol., p. 15.

33. S. Wolfram, 'Cellular automata as models of complexity', Nature, vol. 311, no. 5985, Art. no. 5985 , Oct. 1984 , doi: $10.1038 / 311419 a 0$.

34. S. R. Patel, J. H. Hartwig, and J. E. Italiano, 'The biogenesis of platelets from megakaryocyte proplatelets', J Clin Invest, vol. 115 , no. 12 , pp. $3348-3354$, Dec. 2005 , doi: $10.1172 / \mathrm{JCl} 26891$.

35. R. S. Makar, O. S. Zhukov, M. A. Sahud, and D. J. Kuter, 'Thrombopoietin levels in patients with disorders of platelet production: diagnostic potential and utility in predicting response to TPO receptor agonists', Am J Hematol, vol. 88, no. 12, pp. 1041-1044, Dec. 2013, doi: 10.1002/ajh.23562.

36. K. M. Hoffmeister and H. Falet, 'Platelet clearance by the hepatic Ashwell-Morrell receptor: mechanisms and biological significance', Thromb Res, vol. 141 Suppl 2, pp. S68-72, May 2016, doi: 10.1016/S00493848(16)30370-X.

37. V. V. Bodrova, O. N. Shustova, S. G. Khaspekova, and A. V. Mazurov, 'Platelet reticulated forms, size indexes, and functional activity. Interactions in healthy volunteers', Platelets, pp. 1-6, May 2021, doi: 10.1080/09537104.2021.1922659.

38. L. Hille et al., 'Ultrastructural, transcriptional, and functional differences between human reticulated and non-reticulated platelets', Journal of Thrombosis and Haemostasis, vol. 18, no. 8, pp. 2034-2046, 2020, doi: 10.1111/ jth.14895.

39. S. Chakraborty et al., 'Investigation of the efficacy and safety of eltrombopag to correct thrombocytopenia in moderate to severe dengue patients - a phase II randomized controlled clinical trial', EClinicalMedicine, vol. 29, Dec. 2020, doi: 10.1016/j. eclinm.2020.100624.

40. D. Schmoeller, M. M. Picarelli, T. Paz Munhoz, C. E. Poli de Figueiredo, and H. L. Staub, 'Mean Platelet Volume and Immature
Platelet Fraction in Autoimmune Disorders', Front Med (Lausanne), vol. 4, Sep. 2017, doi: 10.3389/fmed.2017.00146.

41. S. Kovács, Z. Csiki, K. S. Zsóri, Z. Bereczky, and A. H. Shemirani, 'Characteristics of platelet count and size and diagnostic accuracy of mean platelet volume in patients with venous thromboembolism. A systematic review and meta-analysis', Platelets, vol. 30, no. 2, pp. 139-147, Feb. 2019, doi: 10.1080/09537104.2017.1414175.

42. W. Chen and J. Y. Pan, 'Anatomical and Pathological Observation and Analysis of SARS and COVID-19: Microthrombosis Is the Main Cause of Death', Biological Procedures Online, vol. 23, no. 1, p. 4, Jan. 2021, doi: 10.1186/s12575-021-00142-y.

43. S. Yin, M. Huang, D. Li, and N. Tang, 'Difference of coagulation features between severe pneumonia induced by SARS-CoV2 and nonSARS-CoV2', J Thromb Thrombolysis, Apr. 2020, doi: 10.1007/s11239-020-02105-8.

44. N. Ozcelik, S. Ozyurt, B. Y. Kara, A. Gumus, and $U$. Sahin, 'The value of the platelet count and platelet indices in differentiation of COVID-19 and influenza pneumonia', Journal of Medical Virology, vol. 93, no. 4, pp. 22212226, 2021, doi: https://doi.org/10.1002/ jmv. 26645.

45. R. Likic and D. Kuzmanic, 'Severe thrombocytopenia as a complication of acute Epstein-Barr virus infection', Wien Klin Wochenschr, vol. 116, no. 1, pp. 47-50, Jan. 2004, doi: 10.1007/BF03040424.

46. A.-M. Albanyan, M. F. Murphy, J. T. Rasmussen, C. W. Heegaard, and P. Harrison, 'Measurement of phosphatidylserineexposure during storage of platelet concentrates using the novel probe lactadherin: a comparison study with annexin V', Transfusion, vol. 49, no. 1, pp. 99-107, Jan. 2009, doi: 10.1111/j.15372995.2008.01933.x.

47. N. A. Podoplelova, D. Y. Nechipurenko, A. A. Ignatova, A. N. Sveshnikova, and M. A. Panteleev, 'Procoagulant Platelets: Mechanisms of Generation and Action', Hamostaseologie, vol. 41, no. 2, pp. 146153, Apr. 2021, doi: 10.1055/a-1401-2706.

48. J. W. Heemskerk, W. M. Vuist, M. A. Feijge, C. P. Reutelingsperger, and T. Lindhout, 'Collagen but not fibrinogen surfaces induce bleb formation, exposure of phosphatidylserine, and procoagulant activity of adherent platelets: evidence for regulation by protein tyrosine kinase-dependent $\mathrm{Ca2+}$ responses', Blood, vol. 90, no. 7, pp. 26152625, Oct. 1997. 
49. A. C. Kirkpatrick, A. J. Tafur, A. S. Vincent, G. L. Dale, and C. I. Prodan, 'Coated-Platelets Improve Prediction of Stroke and Transient Ischemic Attack in Asymptomatic Internal Carotid Artery Stenosis', Stroke, vol. 45, no. 10, pp. 2995-3001, Oct. 2014, doi: 10.1161/ STROKEAHA.114.006492.

50. M. Schouten, W. J. Wiersinga, M. Levi, and T. van der Poll, 'Inflammation, endothelium, and coagulation in sepsis', Journal of Leukocyte Biology, vol. 83, no. 3, pp. 536-545, 2008, doi: $10.1189 /$ jlb.0607373.

51. S. M. Armstrong, S. Mubareka, and W. L. Lee, 'The lung microvascular endothelium as a therapeutic target in severe influenza', Antiviral Research, vol. 99, no. 2, pp. 113-118, Aug. 2013, doi: 10.1016/j. antiviral.2013.05.003.

52. X. Yang et al., 'Thrombocytopenia and its association with mortality in patients with COVID-19', Journal of Thrombosis and Haemostasis, vol. 18, no. 6, pp. 1469-1472, 2020, doi: $10.1111 /$ jth.14848.

53. J. Thachil et al., 'ISTH interim guidance on recognition and management of coagulopathy in COVID-19', Journal of Thrombosis and Haemostasis, vol. 18, no. 5, pp. 1023-1026, 2020, doi: $10.1111 /$ jth.14810.

54. T. E. Warkentin and J. G. Kelton, 'Temporal Aspects of Heparin-Induced Thrombocytopenia', New England Journal of Medicine, vol. 344, no. 17, pp. 1286-1292, Apr. 2001, doi: 10.1056/NEJM200104263441704.

55. T. E. Warkentin et al., 'Heparin-induced thrombocytopenia in patients treated with low-molecular-weight heparin or unfractionated heparin.', The New England journal of medicine, vol. 332, no. 20, pp. 1330-1335, May 1995, doi: 10.1056/ NEJM199505183322003.

56. C. S. Kow and S. S. Hasan, 'Use of antiplatelet drugs and the risk of mortality in patients with COVID-19: a meta-analysis', J Thromb Thrombolysis, Apr. 2021, doi: 10.1007/ s11239-021-02436-0.

57. J. F.-W. Chan et al., 'Treatment With Lopinavir/ Ritonavir or Interferon- $\beta 1 \mathrm{~b}$ Improves Outcome of MERS-CoV Infection in a Nonhuman Primate Model of Common Marmoset.', The Journal of infectious diseases, vol. 212, no. 12, pp. 1904-1913, Dec. 2015, doi: 10.1093/ infdis/jiv392.

58. I. F.-N. Hung et al., 'Triple combination of interferon beta-1b, lopinavir-ritonavir, and ribavirin in the treatment of patients admitted to hospital with COVID-19: an openlabel, randomised, phase 2 trial.', Lancet
(London, England), vol. 395, no. 10238, pp. 1695-1704, May 2020, doi: 10.1016/S01406736(20)31042-4.

59. M. Edvardsson, M. Oweling, and P. Järemo, 'Small procoagulant platelets in diabetes type 2', Thromb Res, vol. 195, pp. 1-7, Nov. 2020, doi: 10.1016/j.thromres.2020.06.033.

60. M. J. Stotts, J. P. E. Davis, and N. L. Shah, 'Coagulation testing and management in liver disease patients', Curr Opin Gastroenterol, vol. 36, no. 3, pp. 169-176, May 2020, doi: 10.1097/MOG.0000000000000635.

61. E. Vrigkou et al., 'Platelet and coagulation disorders in newly diagnosed patients with pulmonary arterial hypertension', Platelets, vol. 30, no. 5, pp. 646-651, 2019, doi: 10.1080/09537104.2018.1499890.

62. N. Curry, A. Raja, J. Beavis, S. Stanworth, and P. Harrison, 'Levels of procoagulant microvesicles are elevated after traumatic injury and platelet microvesicles are negatively correlated with mortality', J Extracell Vesicles, vol. 3, p. 25625, 2014, doi: 10.3402/jev. v3.25625. 